\title{
WAVE SCATTERING BY A PARTIAL FLEXIBLE POROUS BARRIER IN THE PRESENCE OF A STEP-TYPE BOTTOM TOPOGRAPHY
}

\author{
HAREKRUSHNA BEHERA; TRILOCHAN SAHOO $;$ and CHIU-ON NG
}

\begin{abstract}
A semi-analytic model is presented for oblique wave scattering by a bottom-standing or surface-piercing flexible porous barrier in water of finite depth with a step-type bottom topography. The physical problem is solved using the methods of least-squares and multimode approximation associated with the modified mild-slope equation. Effects on the wave scattering due to bed profile, structural rigidity, compressive force, angle of incidence, barrier length, porosity, and height of the step are examined. The study reveals that under some special conditions, nearly zero/full reflection may occur in the case of wave scattering by a partial flexible porous barrier in the presence of an undulated bottom topography. Further, the study predicts that the Bragg resonance may not occur in the case of wave scattering by a topography of sinusoidal profile. The present study provides insights to help understand how waves are transformed in a marine environment with/without flexible porous barriers in the presence of a bottom topography. The concept and methodology can be generalized to analyze problems of similar nature arising in ocean engineering.
\end{abstract}

Keywords: Wave scattering; step-type bottom; flexible porous barrier; modified mild-slope equation; reflection coefficient.

\section{Introduction}

The interaction of surface water waves with an undulating seabed topography is a problem of fundamental interest in the understanding of wave energy distribution along the coastal region. Apart from semi-analytic methods, numerous numerical models have been developed to study various coastal processes associated with wave motion in the presence of a bottom topography. One of the widely used models for waves over an uneven bottom is based on the mild-slope equation, which was originally derived by Berkhoff [1973]. Smith and Sprinks [1975] later deduced a more precise form of the mild-slope equation similar to that of Berkhoff. Since then, the mild-slope approximation has been extended to incorporate effects due to various kinds of bed topography. Chamberlain and Porter [1995] studied wave scattering by a rippled bed and compared their model with the experimental data

\footnotetext{
*Research Associate, Dept. of Mechanical Engineering, The Univ. of Hong Kong, Pokfulam, Hong Kong. E-mail: hkb.math@gmail.com, behera@hku.hk

${ }^{\dagger}$ Professor, Dept. of Ocean Engineering and Naval Architecture, Indian Institute of Technology Kharagpur, India. E-mail: tsahoo1967@gmail.com, tsahoo@naval.iitkgp.ernet.in

${ }^{\ddagger}$ Professor, Dept. of Mechanical Engineering, The Univ. of Hong Kong, Pokfulam, Hong Kong (corresponding author). E-mail: cong@hku.hk
} 
of Davies and Heathershaw [1984]. Porter and Staziker [1995] derived a modified mild-slope equation (MMSE) to account for slope discontinuities on the bed. They introduced the mass conserving jump conditions into the mild-slope equation using the variational principle. The MMSE with correct interfacial matching conditions has been found to be an efficient model for a bottom slope as steep as 1 .

Meanwhile, a substantial progress has been made on the hydrodynamic analysis of breakwaters for their importance in coastal engineering, environmental protection, recreation and military operations. Compared with barriers that extend from the bottom to the free surface, referred to as complete structures, partial barriers are more preferable in many situations for their lower costs and more environmentalfriendly designs. For example, fixed bottom-standing barriers are often preferred in a marine environment that is dominated by long waves. The main advantage of these bottom-standing barriers is that they do not obstruct marine traffic while attenuating waves in a near-shore region. Likewise, surface-piercing barriers are preferred in deep water regions or regions of poor bed conditions. Work on wave scattering by partial rigid barriers was pioneered by Dean [1945], while work on wave diffraction by an infinite breakwater was started a few years later by Penney and Price [1952]. Some of the other notable contributions on wave diffraction by semi-infinite breakwaters can be found in the book of Wiegel [1964]. Mandal and Chakrabarti [2000] discussed various mathematical tools to deal with water wave scattering by partial rigid barriers of different configurations in water of finite and infinite depths.

In order to reduce wave reflection and loads on coastal structures, perforated walls and wave absorbing structures are used as alternatives for their enhanced ability to dissipate wave energy. Further, for removing unwanted waves during experiments, porous structures are often used as absorbers during physical model tests in laboratories. Isaacson et al. [1998] studied wave interactions with a surfacepiercing partial vertical slotted barrier by using eigenfunction expansion method. Porous barriers have become an important candidate in the art of wave dissipation. Sahoo et al. [2000] studied oblique wave scattering by porous barriers using the least-squares approximation method. Huang et al. [2011] reviewed the hydraulic performance and wave loadings on perforated/slotted coastal structures. The study of oblique wave interaction with porous structure of different configurations are recently studied by Behera and Sahoo [2014].

Besides rigid and permeable structures, flexible and porous structures are also used as breakwaters as these structures are light in weight, economical, reusable and environmental friendly. These types of structures are often deployed for temporary protection of coastal infrastructures/facilities in construction sites, in addition to their use for chord grass seedling, oil spilling and pollution control. Wang and Ren [1993] studied the scattering of small-amplitude waves by a flexible and porous breakwater assuming that the structure is fixed at the seabed and is free near the free surface. Yip et al. [2002] analyzed wave trapping by partial porous and flexible 
barriers. Karmakar et al. [2013] studied the scattering of surface waves by multiple surface-piercing floating membranes. Karmakar and Soares [2014] studied wave transformation due to multiple bottom-standing porous and flexible barriers. Koley et al. [2015a] investigated oblique surface wave scattering by a submerged vertical flexible porous plate for both the cases of finite and infinite water depths using Green's function technique.

In the aforementioned wave-structure interaction problems, the structures are considered to be placed in water of uniform finite or infinite depths. In contrast, there exist only a few studies which have considered wave interaction with vertical/horizontal structures in the presence of an undulated bottom. Using the Galerkin-eigenfunction method, an analytical model was developed by Suh and Park [1995] for wave interaction with a porous barrier near a perforated-wall in the presence of a sloping step. A multi-mode approximation method was extended by Bennetts et al. [2009] to study scattering of flexural-gravity waves with periodic geometries. Bhattacharjee and Guedes Soares [2011] developed an analytical model to study wave interaction with a floating structure near a wall with a stepped bottom. Manam and Kaligatla [2012] studied scattering of flexural and membrane-coupled gravity waves using the modified mild-slope equation. Oblique wave trapping by a thin porous barrier near a wall in the presence of a stepped bed of arbitrary profile was examined by Behera et al. [2015]. Koley et al. [2015b] studied wave interaction with a composite trapezoidal breakwater having an outer perforated layer and an inner rigid core placed on a sloping type topography using the eigenfunction expansion and boundary element method.

The present paper is to look into oblique wave scattering by partial porous and flexible structures in the presence of a step-type bed of various geometrical profiles. Both the cases of bottom-standing and surface-piercing porous barriers are considered under the assumption of small-amplitude water waves and structural response. The modified mild-slope equation along with the methods of eigenfunction expansions and least-squares approximation are used to solve the boundary value problem. Two types of partial structures, namely bottom-standing and surfacepiercing structures, are considered under the assumption that the flow through the porous structures follows Darcy's law. In the case of a bottom-standing barrier, the barrier can be (a) clamped near the seabed and free at the submerged end, or (b) clamped near the seabed and moored at the submerged end. In the case of a surfacepiercing barrier, the barrier is assumed to be (a) clamped near the free surface and free at the submerged end, or (b) clamped near the free surface and moored at the submerged end. To analyze the effect of different types of bed profiles, wave and structural parameters on the wave scattering by the flexible porous barriers, numerical results for the reflection coefficient and barrier deflection are computed and analyzed for different cases. The computational results are validated for their accuracy by comparing with known results in the literature. 


\section{Mathematical Formulation}

In this section, the problem for oblique wave scattering by thin flexible porous barriers of two different configurations is formulated for waves over various types of step-type bottom topography. The two flexible porous barrier configurations are the bottom-standing and surface-piercing barriers as shown in Fig. 1. On the basis of the linearized water wave theory and assuming small-amplitude structural response, the problem is formulated in the three-dimensional Cartesian coordinate system with the $x$ - and $y$-axis being in the horizontal directions and the $z$-axis pointing positive upward. The fluid domain is divided into four sub-domains according to the change of the water depth and the position of the porous barrier. A thin partial flexible porous barrier is kept vertically positioned at a finite distance $L_{1}$ from the edge of a step as shown in Fig. 1. The bed profile is divided into three different parts. The open water region $-\infty<x<0$ is assumed to be of uniform depth $h_{1}$, the undulated bed region extends over $0<x<L_{0}$ and is of variable depth $h(x)$, while the water region beyond $x>L_{0}$ is of uniform depth $h_{2}$. The flexible porous barrier is located at a distance $L_{1}$ beyond $x=L_{0}$, and the fluid domain is thereby divided into four different regions. Further, along the $y$-axis, the fluid domain is assumed to be horizontally extending over $0<y<\infty$. The fluid is assumed to be incompressible, inviscid with motion being irrotational and simple harmonic in time with an angular frequency $\omega$. The problem of obliquely incident waves propagating toward an undulating bathymetry patch is a quasi-3D problem. If the coordinate system is so positioned that the bottom changes only in the $x$-direction, the wave component will not change in its wave number in the $y$-direction, as in Toledo and Agnon [2011]. Thus, the form of the velocity potentials for $j=1,2,3,4$ is given by $\Phi_{j}(x, y, z, t)=\operatorname{Re}\left\{\phi_{j}(x, z) \mathrm{e}^{-\mathrm{i}\left(k_{y} y+\omega t\right)}\right\}$, where $k_{y}=k_{0} \sin \theta$ with $k_{0}$ being the wave number of the incident wave in region 1 and $\theta$ is the incident angle with respect to the $x$-axis.

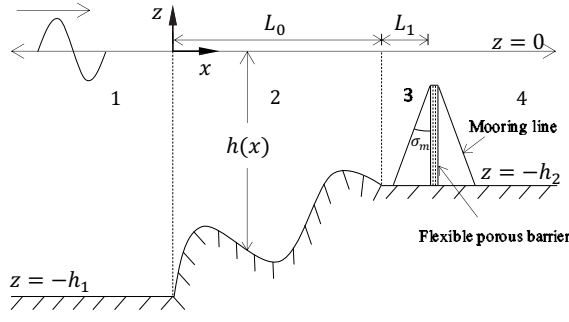

(a)

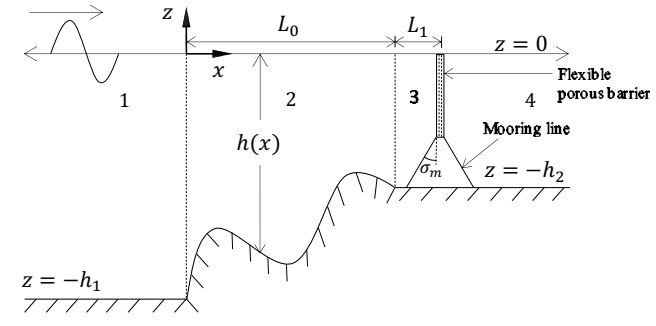

(b)

Fig. 1. Schematic diagram for wave scattering by a (a) bottom-standing, or (b) surface-piercing partial barrier in the presence of bottom undulation. 
Along the vertical $z$-direction, $S_{b}$ and $S_{g}$ represent the regions of the barrier and the gap, respectively, with $b$ being the length of the bottom-standing barrier, and $a$ being the length of the surface-piercing barrier. For the bottom-standing barrier $S_{b}=\left(-h_{2},-h_{2}+b\right)$ and $S_{g}=\left(-h_{2}+b, 0\right)$, while for the surface-piercing barrier $S_{b}=(-a, 0)$ and $S_{g}=\left(-h_{2},-a\right)$. Thus, the spatial velocity potentials $\phi_{j}(x, z)$ for $j=1,2,3,4$ satisfy

$$
\left(\frac{\partial^{2}}{\partial x^{2}}+\frac{\partial^{2}}{\partial z^{2}}-k_{y}^{2}\right) \phi_{j}=0, \quad \text { in the respective fluid domain, }
$$

along with the linearized free-surface boundary condition

$$
\frac{\partial \phi_{j}}{\partial z}-K \phi_{j}=0 \quad \text { on } z=0, \text { for } j=1,2,3,4,
$$

where $K=\omega^{2} / g$ and $g$ is the acceleration due to gravity. Further, $\phi_{j}$, for $j=1,3,4$, satisfies

$$
\frac{\partial \phi_{j}}{\partial z}=0, \quad z=-h_{j} \text { on the uniform bed }
$$

while on the undulated bed, $\phi_{2}$ satisfies

$$
\frac{\partial \phi_{2}}{\partial z}+\frac{d h}{d x} \frac{\partial \phi_{2}}{\partial x}=0 \text { on } z=-h(x)
$$

Assuming that the partial flexible porous barriers are of uniform rigidity, having fine pores, and oscillating in the horizontal direction with displacement of the form $\zeta(y, z, t)=\operatorname{Re}\left\{\xi(z) e^{-\mathrm{i}\left(k_{y} y-\omega t\right)}\right\}$, where $\xi(z)$ is the complex deflection amplitude of the flexible porous barrier and is assumed to be small compared with the water depth. Thus, using Darcy's law for flow past a porous structure, the boundary condition on the flexible porous barrier is given by, as in Behera et al. [2013],

$$
\frac{\partial \phi_{j}}{\partial x}=\mathrm{i} k_{0} G\left(\phi_{3}-\phi_{4}\right)-\mathrm{i} \omega \xi, \quad(j=3,4) \quad \text { on } x=\gamma, \quad z \in S_{b},
$$

where $\gamma=L_{0}+L_{1}$. The complex porous-effect parameter $G$ is of the form $G=$ $G_{r}+\mathrm{i} G_{i}$, and is given by

$$
G=\frac{\delta(f+\mathrm{i} s)}{k_{0} d_{s}\left(f^{2}+s^{2}\right)}
$$

where $\delta, f, s$ and $d_{s}$ are the porosity factor, resistance force coefficient, inertial force coefficient, and thickness of the porous barrier, respectively. Through the real part $G_{r}$ and the imaginary part $G_{i}$, the complex porous-effect parameter $G$ takes into account both the resistance and inertial effects of the porous structure in dissipating wave energy. The resistance force coefficient $f$ and the inertia force coefficient $s$ depend upon the fluid and structure characteristics and are obtained experimentally, 
the details of which are discussed in Li et al. [2006]. The dynamic equation of motion for flexible barrier acted upon by fluid pressure yields, as in Behera et al. [2013],

$$
E I\left(D^{2}-k_{y}^{2}\right)^{2} \xi+Q\left(D^{2}-k_{y}^{2}\right) \xi-m_{s} \omega^{2} \xi=\mathrm{i} \rho \omega\left(\phi_{3}-\phi_{4}\right) \quad \text { for } \quad x=\gamma, \quad z \in S_{b},(7)
$$

where $D \equiv d / d z, E I$ is the uniform flexural rigidity of the barrier, $Q$ is the uniform compressive force acting on the barrier, $m_{s}=\rho_{s} d_{s}$ is the uniform mass per unit length with $\rho_{s}$ being the barrier density, and $\rho$ is the density of water. To keep the flexible barrier in position, the partial barriers are subjected to certain edge conditions at its two ends. In the present study, two different types of edge conditions, namely clamped-moored and clamped-free are considered. Under the clamped-free edge condition, the barrier is assumed to be clamped near the seabed at $\left(\gamma,-h_{2}\right)$ and free near the submerged end at $\left(\gamma,-h_{2}+b\right)$ for a bottom-standing barrier, while in the case of a surface-piercing barrier, it is assumed that the barrier is clamped near the free surface at $(\gamma, 0)$ and is free near the submerged end at $(\gamma,-a)$. Under the clamped-moored edge condition, the barrier is assumed to be clamped near the seabed at $\left(\gamma,-h_{2}\right)$ and moored near the submerged end at $\left(\gamma,-h_{2}+b\right)$ for a bottom-standing barrier, while in the case of a surface-piercing barrier, it is assumed that the barrier is clamped near the free surface at $(\gamma, 0)$ and is moored near the submerged end at $(\gamma,-a)$. The various edge conditions at $z=u$ in terms of the plate deflection are given by

$$
\begin{gathered}
\xi(z)=0, \xi^{\prime}(z)=0 \\
\left(\frac{d^{2}}{d z^{2}}-\nu k_{y}^{2}\right) \xi(z)=0,\left[E I\left\{\frac{d^{2}}{d z^{2}}-(2-\nu) k_{y}^{2}\right\} \frac{d}{d z}+Q \frac{d}{d z}\right] \xi(z)=0 \\
\left(\frac{d^{2}}{d z^{2}}-\nu k_{y}^{2}\right) \xi(z)=0,\left[E I\left\{\frac{d^{2}}{d z^{2}}-(2-\nu) k_{y}^{2}\right\} \frac{d}{d z}+Q \frac{d}{d z}\right] \xi(z)=2 K_{m} \sin ^{2} \sigma_{m} \xi(z) \cdot(
\end{gathered}
$$

Eqs. (8), (9), (10) are the conditions for a clamped edge, free edge and moored edge respectively. Further, in Eqs. (8)-(10), $u$ being $0,-a,-h_{2}+b,-h_{2}$ as appropriate and $\nu$ being the Poisson ratio, $K_{m}=$ mooring line stiffness and $\sigma_{m}=$ the mooring line angle in the static position. The continuity of pressure and normal velocity along the gap yield

$$
\phi_{3}=\phi_{4}, \quad \frac{\partial \phi_{3}}{\partial x}=\frac{\partial \phi_{4}}{\partial x}, \quad \text { on } \quad x=\gamma, \quad z \in S_{g} .
$$

Finally, the radiation conditions are given by

$$
\begin{aligned}
& \phi_{1}=\left(I_{0} \mathrm{e}^{\mathrm{i} p_{0} x}+R_{0} \mathrm{e}^{-\mathrm{i} p_{0} x}\right) f_{0}\left(k_{0}, z\right), \text { as } \quad x \rightarrow-\infty, \\
& \phi_{4}=T_{0} \mathrm{e}^{\mathrm{i} q_{0} x} g_{0}\left(\beta_{0}, z\right), \quad \text { as } \quad x \rightarrow \infty,
\end{aligned}
$$

where $I_{0}$ is the known incident wave amplitude, while $R_{0}$ and $T_{0}$ are unknown constants associated with the amplitudes of the reflected and transmitted waves, 
with $p_{0}=\sqrt{k_{0}^{2}-k_{y}^{2}}, q_{0}=\sqrt{\beta_{0}^{2}-k_{y}^{2}}$. Further, $k_{0}$ and $\beta_{0}$ are the real roots of the dispersion relation in regions 1 and 3 , respectively, with $f_{0}\left(k_{0}, z\right)$ and $g_{0}\left(\beta_{0}, z\right)$ being the associated vertical eigenfunctions.

\section{Method of Solution}

In this section, the method of solution for the mixed boundary value problem associated with the physical problem is briefly described. The undulated bed profile in the interval $\left(0, L_{0}\right)$ is assumed to be a continuously differentiable function having a slope discontinuity at $x=0$ and $x=L_{0}$. In region 1 of uniform depth $h_{1}$, the velocity potential $\phi_{1}$ is written in the form

$$
\phi_{1}(x, z)=I_{0} e^{\mathrm{i} p_{0} x} f_{0}\left(k_{0}, z\right)+\sum_{n=0}^{\infty} R_{n} e^{-\mathrm{i} p_{n} x} f_{n}\left(k_{n}, z\right),
$$

where $f_{n}\left(k_{n}, z\right)=\cosh k_{n}\left(z+h_{1}\right) / \cosh k_{n} h_{1}$ with $p_{n}=\sqrt{k_{n}^{2}-k_{y}^{2}}$ for $n=$ $0,1,2,3, \cdots R_{0}$ is an unknown constant associated with the amplitude of the reflected wave, and $R_{n}$ 's are unknown constants to be determined. Here, $k_{0}$ is the positive real root and $k_{n}$ for $n=1,2,3, \cdots$ are the purely imaginary roots of the dispersion equation $\omega^{2}=g k \tanh k h_{1}$ in $k$. For oblique waves, the wave component does not change in its wave number in the $y$-direction, as has been discussed in Section 2. Thus, in variable-depth region 2 , the velocity potential $\phi_{2}$ is written as

$$
\phi_{2}(x, z)=\sum_{n=0}^{\infty} \psi_{n}(x) W_{n}(h(x), z),
$$

where $\psi_{n}(x)$ s are unknown functions and $W_{n}=\cosh \tilde{k}_{n}(z+h) / \cosh \tilde{k}_{n} h$ with $\tilde{p}_{n}=$ $\sqrt{\tilde{k}_{n}^{2}-k_{y}^{2}}$. The wave number $\tilde{k}_{0}$ is a positive real root and $\tilde{k}_{1}, \tilde{k}_{2}, \tilde{k}_{3}, \cdots$ are purely imaginary roots of the dispersion equation $\omega^{2}=g \tilde{k} \tanh \tilde{k} h$ in $\tilde{k}$. On the other hand, in regions 3 and 4 , the velocity potentials $\phi_{3}$ and $\phi_{4}$ are expressed as

$$
\begin{aligned}
\phi_{3}(x, z) & =\sum_{n=0}^{\infty}\left(A_{n} e^{\mathrm{i} q_{n} x}+B_{n} e^{-\mathrm{i} q_{n} x}\right) g_{n}\left(\beta_{n}, z\right), \\
\text { and } \quad \phi_{4}(x, z) & =\sum_{n=0}^{\infty} T_{n} e^{\mathrm{i} q_{n} x} g_{n}\left(\beta_{n}, z\right)
\end{aligned}
$$

respectively, where $g_{n}\left(\beta_{n}, z\right)=\cosh \beta_{n}\left(z+h_{2}\right) / \cosh \beta_{n} h_{2}$ with $q_{n}=\sqrt{\beta_{n}^{2}-\beta_{y}^{2}}$ for $n=0,1,2, \cdots, A_{n}, B_{n}, T_{n}$ are unknown constants, and $\beta_{0}$ is a positive real root and $\beta_{1}, \beta_{2}, \beta_{3}, \cdots$ are purely imaginary roots of the dispersion equation $\omega^{2}=g \beta \tanh \beta h_{2}$. Hereafter, the infinite series associated with the evanescent modes for the velocity potentials are truncated after $N$ terms. To obtain $\psi_{n}(x)$ in Eq. (15), 
following the procedure of extended modified mild-slope equation (MMSE) as in Porter and Staziker [1995], it is derived that

$$
\begin{aligned}
\frac{d}{d x}\left(a_{n} \frac{d \psi_{n}}{d x}\right)+\sum_{m=0}^{N}\left[\left(b_{m n}\right.\right. & \left.-b_{n m}\right) \frac{d h}{d x} \frac{d \psi_{m}}{d x}+\left\{b_{m n} \frac{d^{2} h}{d x^{2}}\right. \\
& \left.\left.+c_{m n}\left(\frac{d h}{d x}\right)^{2}+d_{m n}-k_{y}^{2} a_{n}\right\} \psi_{m}\right]=0,
\end{aligned}
$$

where

$$
\begin{gathered}
a_{n}(h)=\int_{-h}^{0} W_{n}^{2} d z, b_{m n}(h)=\int_{-h}^{0} W_{n} \frac{\partial W_{m}}{\partial h} d z, c_{m n}(h)=\frac{d b_{m n}}{d h}-\int_{-h}^{0} \frac{\partial W_{m}}{\partial h} \frac{\partial W_{n}}{\partial h} d z \\
d_{m n}(h)=\int_{-h}^{0} W_{n} \frac{\partial^{2} W_{m}}{\partial z^{2}} d z \text { for } n=0,1,2, \cdots, N .
\end{gathered}
$$

Using the continuity of pressure across the interfaces $x=0$ and $x=L_{0}$, the velocity potentials in Eqs. (14)-(16) yield

$$
\left.\begin{array}{l}
\psi_{0}(x)=I_{0} e^{\mathrm{i} p_{0} x}+R_{0} e^{-\mathrm{i} p_{0} x} \\
\psi_{n}(x)=R_{n} e^{-\mathrm{i} p_{n} x}
\end{array}\right\} \text { at } x=0 \text { for } n=1,2, \cdots, N,
$$

and

$$
\psi_{n}(x)=A_{n} e^{\mathrm{i} q_{n} x}+B_{n} e^{-\mathrm{i} q_{n} x} \text { at } x=L_{0} \text { for } n=0,1,2, \cdots, N .
$$

Further, using the conservation of mass across the interface boundaries as in Porter and Staziker [1995] at $x=0$ and $L_{0}$, Eqs. (19) and (20) yield the jump conditions given by

$$
\left.\begin{array}{l}
a_{0} \frac{d \psi_{0}}{d x}+\mathrm{i} p_{0} a_{0} \psi_{0}+h^{\prime} \sum_{m=0}^{N} b_{m 0} \psi_{m}=2 \mathrm{i} p_{0} a_{0} I_{0} \\
a_{n} \frac{d \psi_{n}}{d x}+\mathrm{i} p_{n} a_{n} \psi_{n}+h^{\prime} \sum_{m=0}^{N} b_{m n} \psi_{m}=0
\end{array}\right\} \text { at } x=0+, n=1,2, \cdots, N
$$

and

$$
\begin{gathered}
a_{n} \frac{d \psi_{n}}{d x}-\mathrm{i} q_{n} a_{n} \psi_{n}+h^{\prime} \sum_{m=0}^{N} b_{m n} \psi_{m}=-2 \mathrm{i} a_{n} q_{n} B_{n} e^{-\mathrm{i} q_{n} x} \\
\text { at } x=L_{0}-, n=0,1,2, \cdots, N .
\end{gathered}
$$

Using the orthogonal characteristics of the eigenfunctions $g_{n}(\beta, z)$, substituting the velocity potentials $\phi_{3}$ and $\phi_{4}$ as in Eqs. (16) and (17) into the condition for continuity of velocity as in Eqs. (5) and (11) along the flexible barrier and the gap at $x=$ $L_{0}+L_{1}=\gamma$, it is derived that

$$
A_{n}-B_{n} e^{-2 \mathrm{i} q_{n} \gamma}=T_{n}, \quad \text { for } \quad n=0,1,2, \cdots
$$


Next, from Eqs. (7), (16) and (17), the plate deflection $\xi(z)$ is obtained as

$$
\xi(z)=\sum_{m=1}^{4} C_{m} \tilde{f}_{m}(z)+\sum_{n=0}^{\infty} v_{n}\left(A_{n}-T_{n}\right) e^{\mathrm{i} q_{n} \gamma} g_{n}(z), \quad \text { for } \quad z \in S_{b},
$$

where

$$
\begin{aligned}
& v_{n}=\frac{2 \mathrm{i} \rho \omega}{E I q_{n}^{4}+Q q_{n}^{2}-m_{s} \omega^{2}}, \quad \tilde{f}_{1}(z)=\frac{\cosh \tau_{1} z}{\cosh \tau_{1} h_{2}}, \quad \tilde{f}_{2}(z)=\frac{\sinh \tau_{2} z}{\sinh \tau_{2} h_{2}}, \\
& \tilde{f}_{3}(z)=\frac{\cos \tau_{3} z}{\cos \tau_{3} h_{2}}, \quad \tilde{f}_{4}(z)=\frac{\sin \tau_{4} z}{\sin \tau_{4} h_{2}}
\end{aligned}
$$

with $C_{m}$ being unknown constants to be determined, $\tau_{n}$ 's being the roots of the characteristic equation $E I\left(\tau_{n}^{2}-k_{y}^{2}\right)^{2}+Q\left(\tau_{n}^{2}-k_{y}^{2}\right)-m_{s} \omega^{2}=0$ with $\tau_{n}=i \tau_{n}$ for $n=3$, 4. Substituting for $\xi(z)$ from Eq. (24) in Eq. (5), a series relation for the unknowns is obtained as

$$
\sum_{n=0}^{\infty}\left(A_{n} r_{n}+J_{n} T_{n}\right) g_{n}(z)+\mathrm{i} \omega \sum_{m=1}^{4} C_{m} \tilde{f}_{m}(z)=0, \quad \text { for } \quad z \in S_{b}
$$

where $J_{n}=\left(\mathrm{i} q_{n}+2 \mathrm{i} k_{0} G-\mathrm{i} \omega v_{n}\right) e^{\mathrm{i} q_{n} \gamma}$ and $r_{n}=\mathrm{i}\left(v_{n} \omega-2 k_{0} G\right) e^{\mathrm{i} q_{n} \gamma}$. Further, using the continuity of velocity potential of $\phi$ as in Eq. (11) and the relations in Eq. (23), another series relation is obtained as

$$
\sum_{n=0}^{\infty}\left(A_{n}-T_{n}\right) e^{\mathrm{i} q_{n} \gamma} g_{n}(z)=0, \quad \text { for } \quad z \in S_{g}
$$

The series relations in Eqs. (25) and (26) are satisfied in disjoint intervals and are referred to as dual series relations. These dual series relations in Eqs. (25) and (26) are rewritten as

$$
D(z)=\sum_{n=0}^{\infty}\left\{\ell_{1 n}(z)+T_{n} \ell_{2 n}\right\}=0, \quad-h_{2}<z<0,
$$

where

$$
\begin{aligned}
& \ell_{1 n}(z)= \begin{cases}A_{n} e^{\mathrm{i} q_{n} \gamma} g_{n}(z), & \text { for } z \in S_{g}, \\
\sum_{m=1}^{4} C_{m} \tilde{f}_{m}(z)+A_{n} r_{n} g_{n}(z), & \text { for } z \in S_{b},\end{cases} \\
& \ell_{2 n}(z)= \begin{cases}-e^{\mathrm{i} q_{n} \gamma} g_{n}(z), \text { for } z \in S_{g}, \\
J_{n} g_{n}(z), & \text { for } z \in S_{b} .\end{cases}
\end{aligned}
$$

In Eq. (27), the $N$-th partial sum of the series $D(z)$ is given by

$$
D_{N}(z)=\sum_{n=0}^{N}\left\{\ell_{1 n}(z)+T_{n} \ell_{2 n}(z)\right\}=0, \quad-h_{2}<z<0 .
$$


Using the least-squares approximation method, Eqs. (27) and (30) yield

$$
\int_{-h_{2}}^{0} D_{M}^{*}(z) D_{T_{n}}(z) d z=0, \quad \text { for } \quad n=0,1,2,3, \cdots, M,
$$

where $*$ denotes the complex conjugate and $D_{T_{n}}(z)$ is the derivative of $D_{M}(z)$ with respect to $T_{n}$. Using Eq. (30) in Eq. (31), a system of linear equations is obtained as follows:

$$
\sum_{m=0}^{N} T_{m}^{*} X_{m n}=\tilde{d}_{n}, \quad \text { for } \quad n=0,1,2, \cdots, M
$$

with

$$
\begin{array}{r}
\tilde{d}_{n}=\int_{-h_{2}}^{0} \ell_{1 n}^{*}(z) \ell_{1 n}(z) d z, \quad X_{m n}=\int_{-h_{2}}^{0} \ell_{2 n}^{*}(z) \ell_{2 n}(z) d z \\
\text { for } m, n=0,1,2, \cdots, M .
\end{array}
$$

For the determination of the unknown function $\psi_{n}$ for specific bed profile $h(x)$, Eq. (18) is solved numerically using the Runge-Kutta method. The computed results for $\psi_{n}$ 's are used in Eqs. (19)-(21) and (32) to obtain a system of $6 N$ equations. Another four linear equations are obtained from the edge conditions as in Eqs. (8) and (10). The set of $6 N+4$ linear equations are then solved for the various physical quantities of interest. The computed results are discussed in the next section.

\section{Numerical Results and Discussion}

In this section, results generated by MATLAB programs which have been developed to solve the system of equations described above are presented. The aim is to investigate the effects of various wave and structural parameters on the wave reflection, plate deflection and hydrodynamic forces on the porous plate. Unless stated otherwise, the physical parameters are kept fixed at the following values: wave period $T=8 \mathrm{~s}$, acceleration due to gravity $g=9.81 \mathrm{~m} / \mathrm{s}^{2}$, depth ratio $h_{2} / h_{1}=0.5, L_{0} / h_{1}=0.5, L_{1} / h_{1}=3, \vartheta=E I /\left(\rho g h_{2}^{4}\right)=0.1, \tau=Q /\left(\rho g h_{2}^{2}\right)=0.1$, $v=m_{s} /\left(\rho h_{2}\right)=0.1, \nu=0.3, a / h_{2}=b / h_{2}=0.5, \sigma_{m}=45^{\circ}, K_{m}=10^{3} \mathrm{~N} \mathrm{~m}^{-1}$, $k_{0} h_{1}=0.5$ and $\theta=30^{\circ}$. The reflection coefficient $K_{r}$ is defined as

$$
K_{r}=\left|R_{0} / I_{0}\right| \text {. }
$$

In the subsequent discussions, numerical results for various bed profiles are analyzed separately.

\subsection{Bed profile type 1}

Here, the bed profiles shown in Figs. 2(a) and (b) are considered using the bed function $h(x)$ as follows:

$$
h(x)=h_{1}-\tilde{b}\left\{1-\alpha\left(1-x / L_{0}\right)^{2}+(\alpha-1)\left(1-x / L_{0}\right)\right\}, \quad 0<x<L_{0},
$$




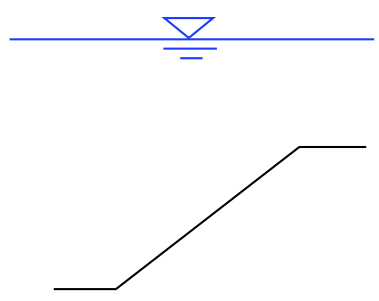

(a) Type $1(\alpha=0)$

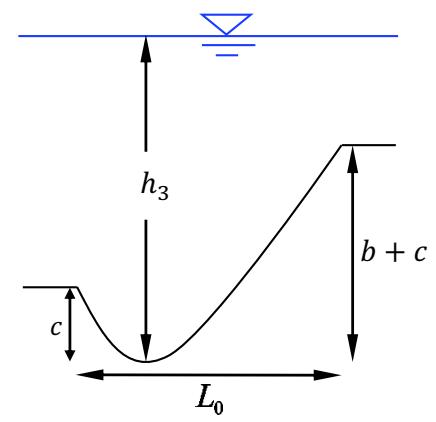

(c) Type 2

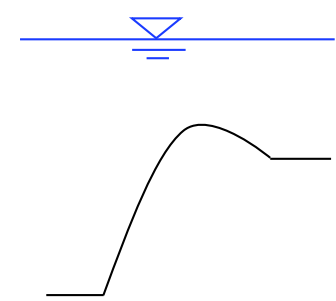

(b) Type $1(\alpha>1)$

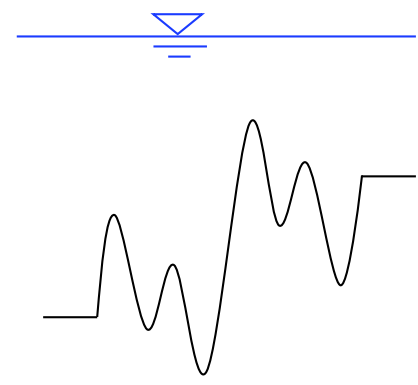

(d) Type 3

Fig. 2. Schematic diagram of different types of bed profiles (the top line being the static water surface).

where $\tilde{b}=h_{1}-h_{2}$. In Eq. (35) for the bed function $h(x), \alpha=0$ refers to the sloping step-type bed as shown in Fig. 2(a). On the other hand, for $\alpha>1$, protrusion occurs above the depth $h_{2}$ as in Fig. 2(b). Other forms of the bed profile can be considered with change in $\alpha$; see Behera et al. [2015]. In Figs. 3(a) and 3(b), the reflection and transmission coefficients versus the non-dimensional wave number $k_{0} h_{1}$ are plotted for a fully extended bottom-standing rigid porous barrier. From Fig. 3(a), it is found that the curves of $K_{r}$ and $K_{t}$ obtained by the present theory for uniform bed are in close agreement with the experimental and theoretical results of Li et al. [2006]. These comparisons should give a check on the correctness of the results presented in this paper. On the other hand, the sloping angle decreases with an increase in the depth ratio $h_{2} / h_{1}$. Thus, the reflection coefficient decreases whereas the transmission coefficient increases as a result of the increase of the depth ratio $h_{2} / h_{1}$, as shown in Fig. 3(b). Moreover, the reflection and transmission coefficients follow certain oscillatory pattern with a decrease in the depth ratio $h_{2} / h_{1}$.

In Figs. 4(a) and 4(b), the reflection and transmission coefficients versus the nondimensional wave number $k_{0} h_{1}$ are plotted for a bottom-standing porous barrier for 
different values of the mooring line angle $\sigma_{m}$ and stiffness $K_{m}$, respectively. Fig. 4(a) reveals that wave reflection is larger and wave transmission is smaller when the barrier is clamped near the seabed and moored at the submerged end, when compared with that under the clamped-free edge condition. This is due to the fact that more waves get transmitted through the flexible porous barrier when the barrier is under the clamped-free edge condition. Further, the wave reflection increases, and the wave transmission decreases, with an increase in the mooring angle $\sigma_{m}$. As the stiffness of the mooring line decreases, the flexible barrier deflects to a greater extent. Thus, with an increase in the mooring line stiffness $K_{m}$, the reflection coefficient decreases as shown in Fig. 4(b). The reverse pattern is observed for the transmission coefficient.

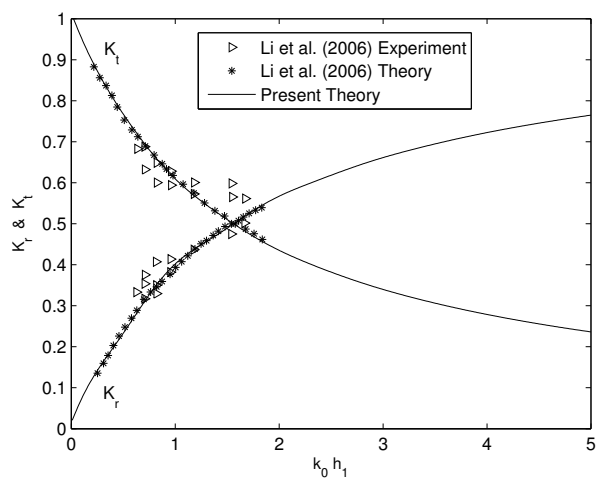

(a)

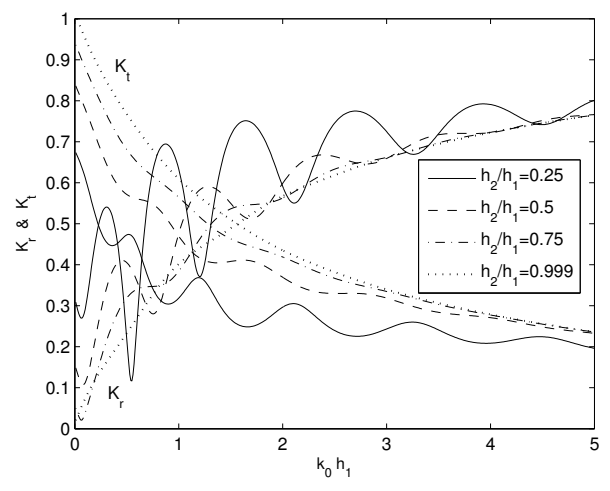

(b)

Fig. 3. The reflection and transmission coefficients as functions of $k_{0} h_{1}$ : (a) a comparison between present results with the theoretical and experimental results of Li et al. [2006] for $h_{2} / h_{1}=1$, and (b) effect of the depth ratio $h_{2} / h_{1}$ in the case of a rigid porous barrier with $b / h_{2}=1, \delta=0.2$, $f=9, s=1, d_{s}=0.0286, \alpha=0, \theta=0^{\circ}, \sigma_{m}=0^{\circ}, \tau=0$ and $\vartheta=20$.

In Figs. 5(a) and 5(b), the reflection coefficient versus the non-dimensional wave number $k_{0} h_{1}$ are plotted for a bottom-standing porous barrier for different values of depth ratio $h_{2} / h_{1}$ and porous-effect parameter $G$, respectively. Fig. 5(a) reveals that the wave reflection decreases with an increase in the depth ratio. However, for $h_{2} / h_{1}=1$, the pattern in the reflection coefficient is similar to the observation made previously in Koley et al. [2015a] for wave scattering by a bottom-standing partial flexible porous barrier in water of uniform depth. Further, Fig. 5(a) reveals that for $k_{0} h_{1} \approx 0$, zero reflection occurs by a flexible porous barrier in the case of uniform water depth. However, when waves propagate by a flexible porous barrier in the presence of a sloping bed, for $k_{0} h_{1} \approx 0$, non-zero wave reflection occurs. On the other hand, for $h_{2} / h_{1}<1$, oscillatory patterns in wave reflection are observed 


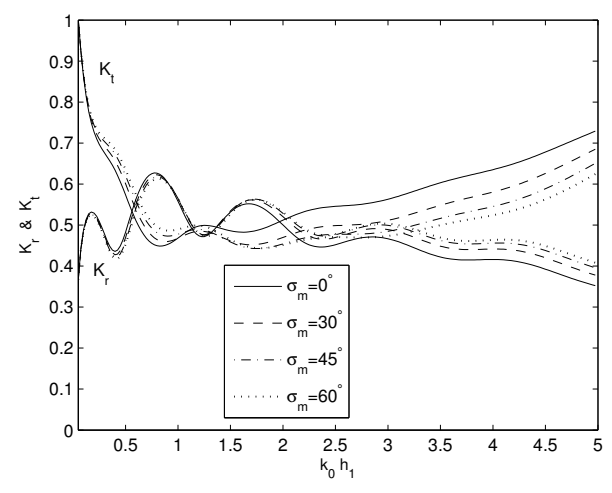

(a)

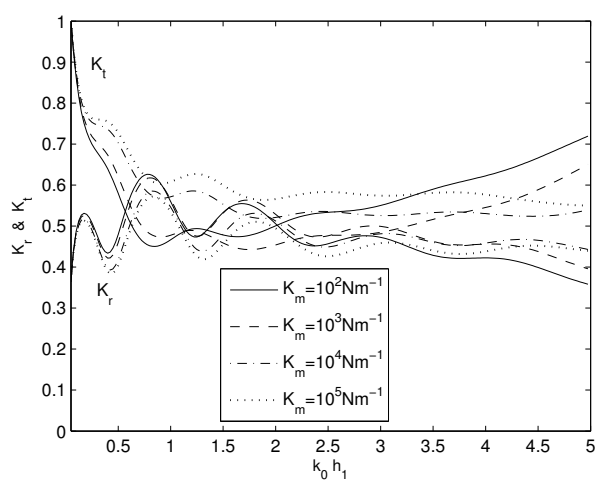

(b)

Fig. 4. Effect of the mooring line (a) angle $\sigma_{m}$, and (b) stiffness $K_{m}$ on the reflection and transmission coefficients as functions of the non-dimensional incident wave number $k_{0} h_{1}$, with (a) $K_{m}=10^{3} \mathrm{~N} \mathrm{~m}^{-1}$, and (b) $\sigma_{m}=45^{\circ}$, for $h_{2} / h_{1}=0.5, G=0.5+0.5 \mathrm{i}, \theta=30^{\circ}$, and $\alpha=0$.

for smaller values of the wave number $k_{0} h_{1}$ which diminishes for $k_{0} h_{1}>\pi$. This demonstrates that wave reflection follows certain steady pattern in the case of deep water waves and is highly oscillatory in case of long waves. Moreover, it may be noted that the oscillatory pattern in the wave reflection diminishes with an increase in the depth ratio $h_{2} / h_{1}$, corresponding to a steeper slope of the step profile. From Fig. 5(b), it is observed that the reflection coefficient decreases with an increase in the absolute value of the porous-effect parameter $G$. Further, it is observed that full reflection occurs in the case of an impermeable barrier, i.e., $G=0$, for $k_{0} h_{1}=0.4$. The occurrence of this full reflection is possibly due to the combined effect of an impermeable barrier and a sloping step. On the other hand, nearly zero reflection occurs for $k_{0} h_{1}=1.3$ with $G=5+5$ i. The occurrence of nearly zero reflection is a result of the destructive interference of the incoming and reflected waves in the case of wave scattering by a flexible porous barrier in the presence of a sloping step on the bed.

In Figs. 6(a) and 6(b), the reflection coefficient versus the non-dimensional wave number $k_{0} h_{1}$ are plotted for a bottom-standing porous barrier for different values of $\alpha$ and length of the porous barrier $b / h_{2}$, respectively. In these figures, the general patterns of the wave reflection are similar to those shown in Figs. 5(a) and 5(b). Fig. 6 (a) reveals that the wave reflection increases with an increase in $\alpha$. This is expected since a protrusion on the bottom will tend to reflect waves back. Further, Fig. 6(b) reveals that the reflection coefficient increases with an increase in the length of the porous barrier $b / h_{2}$ for smaller value of $k_{0} h_{1}$. In contrast, wave reflection will decrease with an increase in $b / h_{1}$ for larger values of $k_{0} h_{1}$.

In Figs. 7(a) and 7(b), the reflection coefficient versus the non-dimensional wave number $k_{0} h_{1}$ are plotted in the case of a bottom-standing porous barrier for different 
14

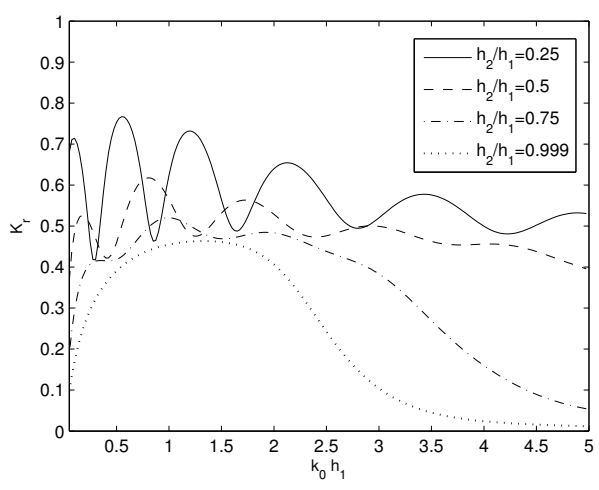

(a)

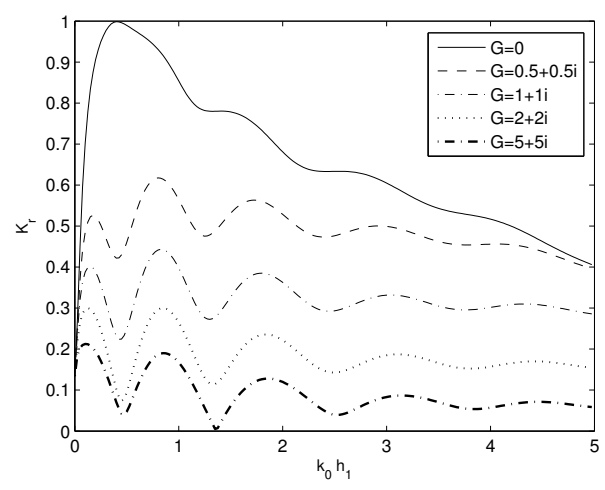

(b)

Fig. 5. Effect of the (a) depth ratio $h_{2} / h_{1}$, and (b) porous-effect parameter $G$ on the reflection coefficient as a function of the non-dimensional incident wave number $k_{0} h_{1}$, with (a) $G=0.5+0.5 \mathrm{i}$, and (b) $h_{2} / h_{1}=0.5$, for $\theta=30^{\circ}$, and $\alpha=0$.

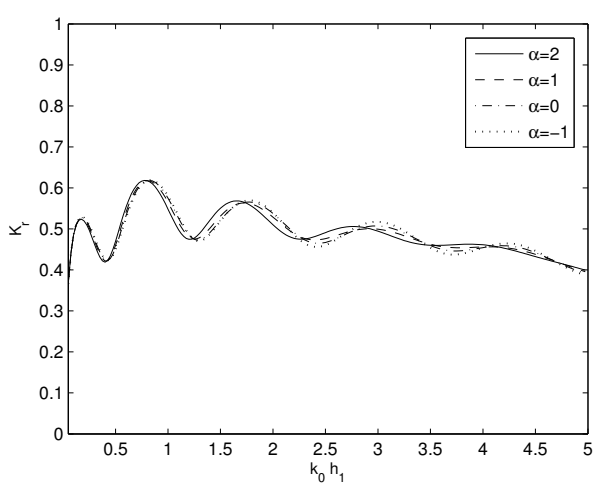

(a)

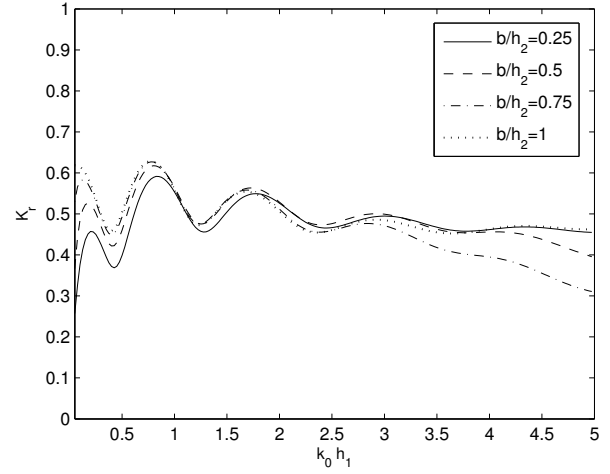

(b)

Fig. 6. Effect of the (a) bed profile constant $\alpha$, and (b) length of the porous barrier $b / h_{2}$ on the reflection coefficient as a function of the non-dimensional incident wave number $k_{0} h_{1}$, with (a) $b / h_{2}=0.5$, and (b) $\alpha=0$, for $G=0.5+0.5 \mathrm{i}$, and $\theta=30^{\circ}$.

values of non-dimensional flexural rigidity $\vartheta$ and compressive force $\tau$, respectively. These figures depict that the general patterns of the reflection coefficients are similar to those shown in Figs. 5 and 6. Fig. 7(a) depicts that the reflection is larger in the case of a stiffer structure. Similar observations are found in Karmakar and Soares [2014]. This may be due to the fact that as structural rigidity increases flexible structure tends to behave like a rigid structure. Also, Fig. 7(b) reveals that the wave reflection increases with an increase in the compressive force acting on the plate for smaller values of $k_{0} h_{1}$. This is similar to wave diffraction by a floating 
flexible plate under a compressive force as in Mohapatra et al. [2013]. This is due to the fact that for smaller values of wave number $k_{0} h_{1}$, more wave energy which concentrates near the free surface is reflected by the structure for higher compressive force. However, the wave reflection decreases with an increase in the compressive force for larger values of $k_{0} h_{1}$ for wave propagation in uniform water depth.

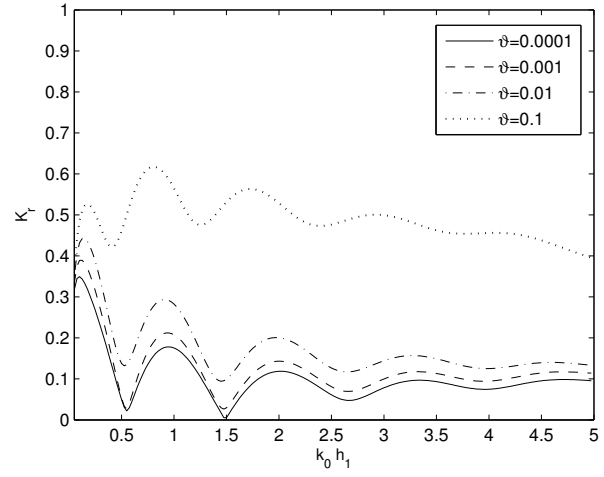

(a)

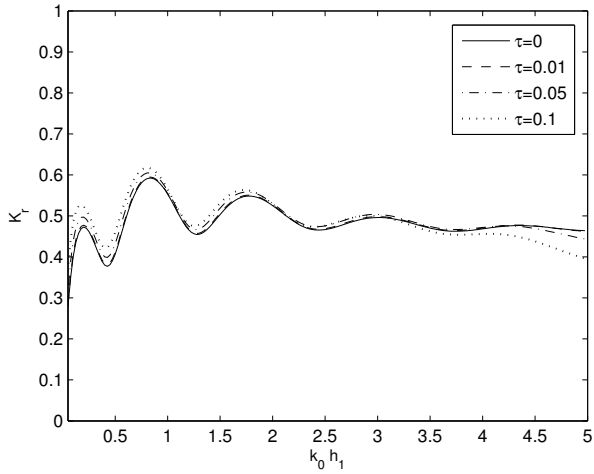

(b)

Fig. 7. Effect of the (a) non-dimensional flexural rigidity $\vartheta$, and (b) compressive force $\tau$ on the reflection coefficient as a function of the non-dimensional incident wave number $k_{0} h_{1}$, with (a) $\tau=0.1$, and (b) $\vartheta=0.1$, for $\alpha=0, G=0.5+0.5 \mathrm{i}$ and $\theta=30^{\circ}$.

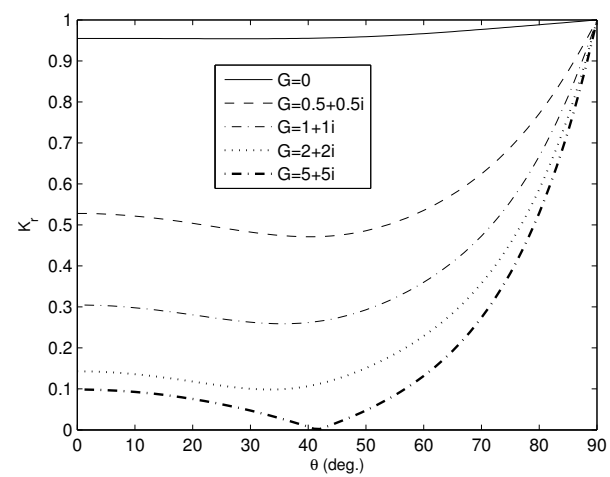

(a)

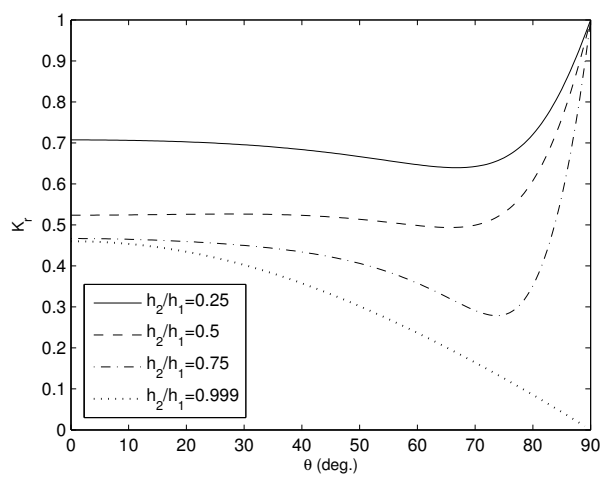

(b)

Fig. 8. Effect of the (a) porous-effect parameter $G$, and (b) depth ratio $h_{2} / h_{1}$ on the reflection coefficient as a function of the incident angle $\theta$, with (a) $h_{2} / h_{1}=0.5$, and (b) $h_{2} / h_{1}$, for $G=$ $0.5+0.5 \mathrm{i}, k_{0} h_{1}=0.5$, and $\alpha=0$.

In Figs. 8(a) and 8(b), the reflection coefficients $K_{r}$ versus the incident angle 
$\theta$ are plotted in the case of a bottom-standing porous barrier for different values of porous-effect parameter $G$ and depth ratio $h_{2} / h_{1}$, respectively. Fig. 8(a) depicts that the reflection coefficient decreases with an increase in the absolute value of the porous-effect parameter $G$. The decrease in wave reflection results from an increased transmission of wave energy through the porous barrier for larger values of $|G|$. Further, the wave reflection attains zero minimum in the case of $G=5+5 \mathrm{i}$ for $\theta=41^{\circ}$. The minimum in wave reflection at a certain angle of incidence may be due to destructive interference of the incoming and reflected waves in the presence of a step-type bottom, which is similar to wave past a submerged rectangular impermeable/permeable structure as in Abul-Azm [1994] and Losada et al. [1996]. In addition, Fig. $8(\mathrm{~b})$ reveals that wave reflection decreases with an increase in the depth ratio $h_{2} / h_{1}$ having a minimum at a certain angle of incidence $\theta$. After attaining the minimum, with further increase in the angle of incidence, the wave reflection increases to reach full reflection at $90^{\circ}$. It is also noted that wave reflection decreases to zero with an increase in the angle of incidence $\theta$ in the case of a uniform depth, which is similar to oblique wave scattering by porous barriers in uniform depth as in Sahoo et al. [2000]. The variation in wave reflection for an oblique angle $\theta$ approaching $90^{\circ}$ is possibly due to the diffraction and shoaling of gravity waves in the presence of a bottom undulation; these details are, however, beyond the scope of the present study.

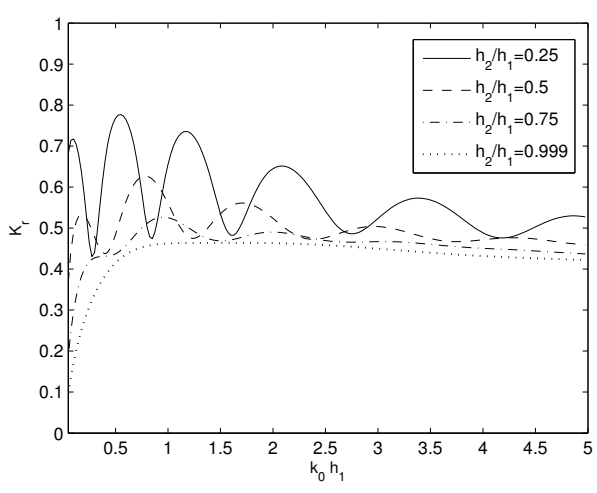

(a)

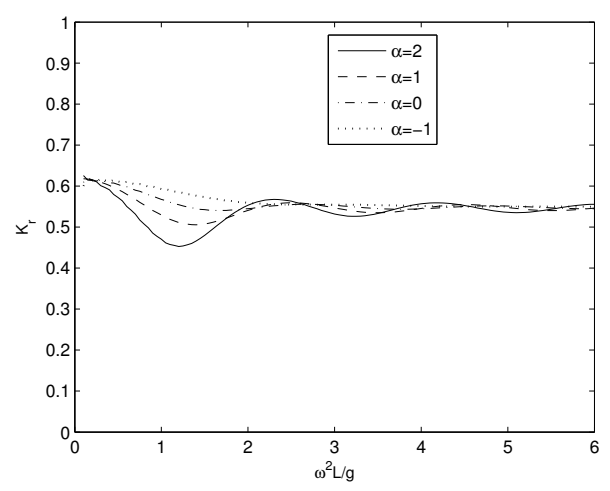

(b)

Fig. 9. Effect of the (a) depth ratio $h_{2} / h_{1}$, and (b) bed profile constant $\alpha$ on the reflection coefficient as a function of the non-dimensional incident wave number $k_{0} h_{1}$ and slope length $w^{2} L_{0} / g$, respectively, in case of a surface-piercing barrier, for (a) slope length $L_{0} / h_{1}=0.5$ and $\alpha=0$, and (b) $w^{2} / g=0.6$, and $h_{2} / h_{1}=0.5$.

In Figs. 9(a) and 9(b), the reflection coefficient versus the non-dimensional wave number $k_{0} h_{1}$ and slope length $w^{2} L_{0} / g$ are plotted, respectively, in the case of a surface-piercing porous barrier for different values of (a) depth ratio $h_{2} / h_{1}$, and (b) 
$\alpha$. In Fig. 9(a), the pattern in wave reflection except $h_{2} / h_{1} \approx 1$ is quite similar to that of a bottom-standing porous barrier as discussed in Figs. 5(a). Further, a comparison of Figs. 5(a) and 9(a) reveals that for larger values of $k_{0} h_{1}$, the wave reflection is smaller for the bottom-standing barrier compared with the surface-piercing plate. This is a consequence of the fixed edge condition near the free surface for a surfacepiercing barrier, which does not allow plate deflection near the free surface, leading to larger wave energy reflection. Fig. 9(a) shows that for wave scattering by flexible barriers over a uniform depth, initially wave reflection increases with an increase in the wave number $k_{0} h_{1}$ and attains a steady state for larger values of the wave number $k_{0} h$. Similar observations were made before by Koley et al. [2015a] for wave scattering by a porous and flexible plate in water of uniform depth. Also, in Fig. 9(b), the pattern in wave reflection is similar to that of the modified mild-slope equation as in Porter and Staziker [1995]. In general, the reflection coefficient decreases in an oscillatory pattern with an increase in the slope length. The amplitude of the oscillatory pattern of the reflection coefficient decreases with an decrease in $\alpha$, an effect due to the protrusion of the step profile.

\subsection{Bed profile type 2}

Here, the bed profile as shown in Fig. 2(c) is considered, with the bed function $h(x)$ given by

$$
\begin{aligned}
h(x)= & h_{3}-\left(h_{3}-h_{2}\right)\left[1-\tilde{a}\left(1-x / L_{0}\right)^{4}+\left\{2 \tilde{a}+2\left(1-\lambda_{t}\right)\right\}\left(1-x / L_{0}\right)^{3}\right. \\
& \left.-\left\{\tilde{a}+3\left(1-\lambda_{t}\right)\right\}\left(1-x / L_{0}\right)^{2}\right],
\end{aligned}
$$

where $\tilde{c}=h_{3}-h_{1}, \lambda_{t}=\tilde{c} /(\tilde{b}+\tilde{c})$ and $\tilde{a}$ is real and positive, and satisfies the relation

$$
16 \tilde{a}^{3}-\left\{\tilde{a}+3\left(1-\lambda_{t}\right)\right\}^{3}\left\{\tilde{a}-\left(1-\lambda_{t}\right)\right\}=0 .
$$

In this case, there is a depression in the bed profile for $0<x<L_{0}$. In the following, numerical results for bottom-standing and surface-piercing barriers are analyzed separately.

In Figs. 10(a) and 10(b), the reflection and transmission coefficients versus the non-dimensional wave number $k_{0} h_{1}$ are plotted for different values of the depth ratio $h_{2} / h_{1}$, respectively, in the case of a bottom-standing flexible porous barrier. Fig. 10(a) depicts that the general patterns of the reflection coefficients are similar to the results associated with the sloping bed profiles as in Fig. 5(a). However, an opposite trend is observed for the transmission coefficients, as shown in Fig. 10(b). Also, owing to the depression of the step profile as shown in Fig. 2(d), the wave reflection pattern is more oscillatory compared to that shown in Fig. 4(a).

In Figs. 11(a) and 11(b), the reflection and transmission coefficients versus the non-dimensional wave number $k_{0} h_{1}$ are plotted for different values of depth ratio $h_{3} / h_{1}$ and compressive force $\tau$, respectively in the case of a bottom-standing flexible porous barrier. Fig. 11(a) reveals that the amplitudes of oscillation in the 


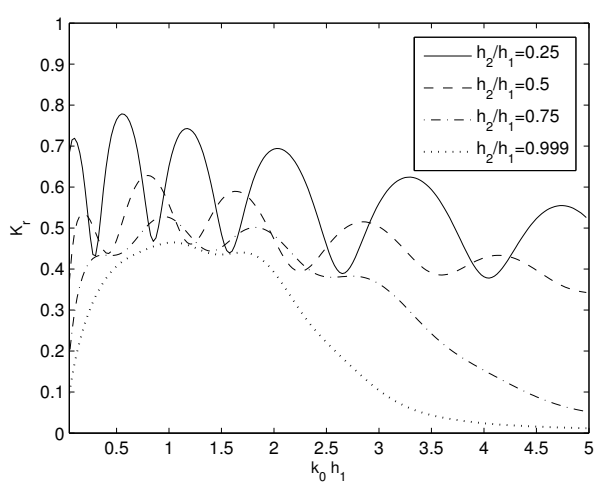

(a)

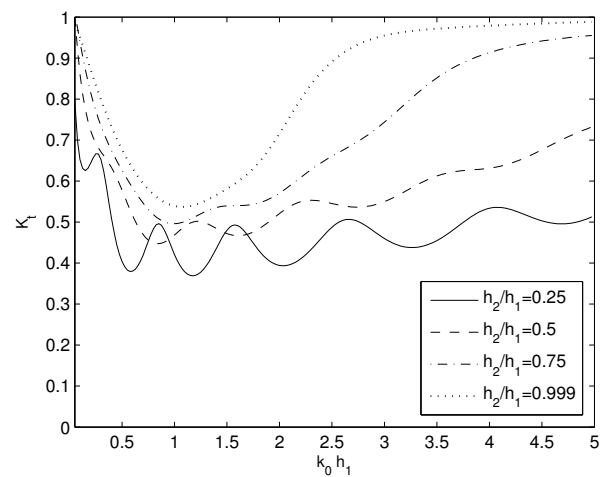

(b)

Fig. 10. Effect of the depth ratio $h_{2} / h_{1}$ on the (a) reflection and (b) transmission coefficients as functions of the non-dimensional wave number $k_{0} h_{1}$, with $h_{3} / h_{1}=1.4$, and $\theta=30^{\circ}$.

reflection and transmission coefficients are larger with an increase in the bottom depression $h_{3} / h_{1}$ for $1 \leq k_{0} h_{1} \leq 4$, beyond which the wave reflection and transmission are independent of the depth ratio $h_{3} / h_{1}$. Moreover, irrespective of the depression, maximum wave reflection and transmission occur for $1 \leq k_{0} h_{1} \leq 4$. From Fig. 11(b), it is observed that with an increase in the compressive force $\tau$, the reflection coefficients increase whereas transmission coefficients decrease for smaller values of the wave number $k_{0} h_{1}$ corresponding to longer waves. A comparison with Fig. 7(b) reveals that the scattering coefficients are opposite in nature with change in the compressive force. Moreover, Fig. 11(b) reveals that for larger values of wave number $k_{0} h_{1}$, corresponding to deeper water waves, the reflection coefficient decreases and transmission coefficient increases with an increase in $\tau$.

In Figs. 12(a) and 12(b), the reflection coefficient versus the normalized distance between the porous barrier and step $L_{1} / \lambda_{1}$ are plotted for different values of (a) porous-effect parameter $G$, and (b) step length $L_{0} / h_{1}$, respectively, in the case of a bottom-standing flexible porous barrier. The figures show that the general pattern of the wave reflection coefficients varies periodically with an increase in $L_{1} / h_{1}$. Fig. 12(a) depicts that the wave reflection decreases with an increase in the absolute value of the porous-effect parameter $G$. However, with an increase in the absolute value of the porous-effect parameter, the amplitude of the oscillatory pattern in wave reflection increases. Fig. 12(b) also shows that the amplitude of the oscillatory pattern in wave reflection increases with an increase in the length of the step $L_{0} / h_{1}$. Further, there is a left shift in the amplitude of the oscillatory pattern in wave reflection with an increase in the length $L_{0} / h_{1}$ of the step profile. This may be due to the decrease in the sloping angle of the step leading to a change of the phase of the reflected waves.

In Figs. 13(a) and 13(b), the reflection coefficient versus the non-dimensional 


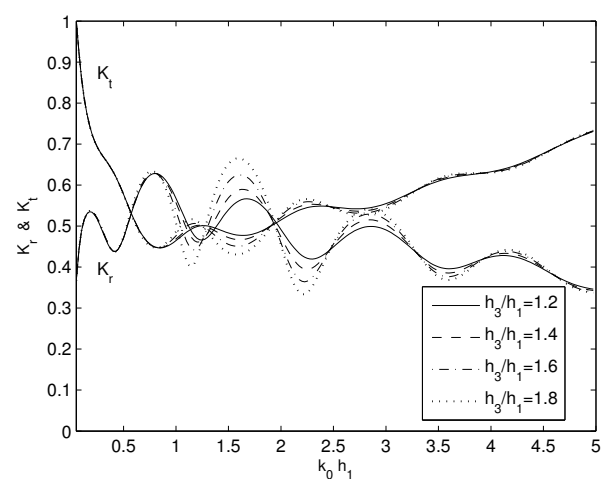

(a)

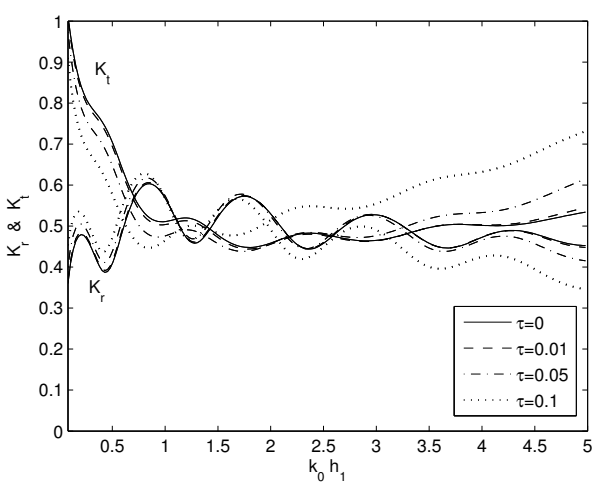

(b)

Fig. 11. Effect of the (a) depth ratio $h_{3} / h_{1}$, and (b) compressive force $\tau$ on the reflection and transmission coefficients as functions of the non-dimensional incident wave number $k_{0} h_{1}$, with (a) $\tau=0.1$ and (b) $h_{3} / h_{1}=1.4$, for $L_{0} / h_{1}=0.5, G=0.5+0.5 \mathrm{i}, \theta=30^{\circ}, h_{2} / h_{1}=0.5$, and $h_{3} / h_{1}=1.4$.

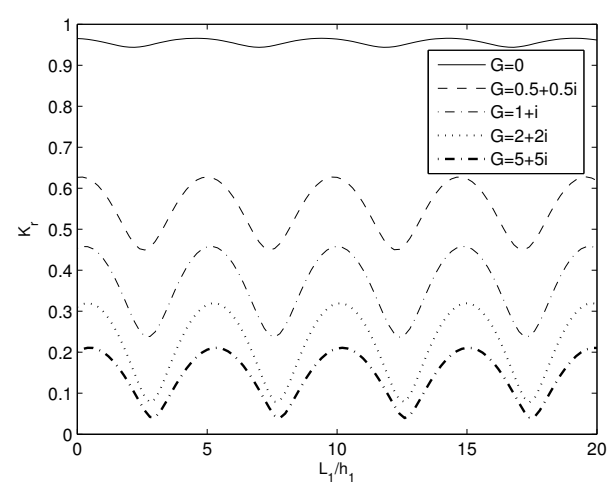

(a)

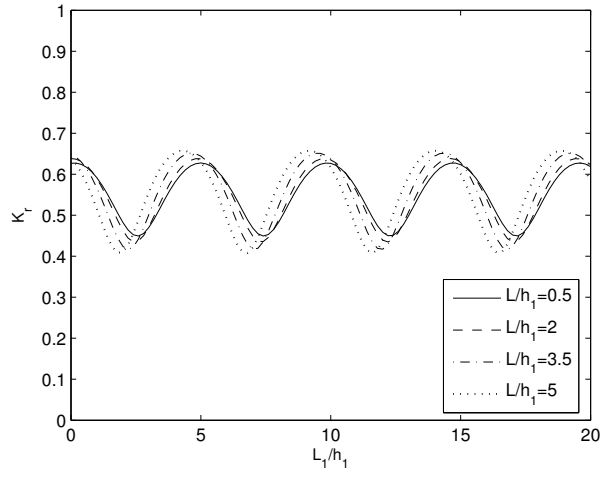

(b)

Fig. 12. Effect of the (a) porous-effect parameter $G$, and (b) step length $L_{0} / h_{1}$ on the reflection coefficient as a function of the normalized distance between the porous barrier and step $L_{1} / \lambda_{1}$, with (a) $L_{0} / h_{1}=0.5$, and (b) $G=0.5+0.5 \mathrm{i}$, for $\theta=30^{\circ}, h_{2} / h_{1}=0.5$, and $h_{3} / h_{1}=1.4$.

flexural rigidity $\vartheta$ are shown for a (a) uniform depth, and (b) step-type bed with $h_{2} / h_{1}=0.5$ for different values of $G$, respectively, in the case of a bottom-standing flexible porous barrier having a depression on the bed. Both the figures depict that the curve of the wave reflection is more oscillatory for smaller values of the nondimensional flexural rigidity $\vartheta$ and the oscillatory pattern diminishes for larger values of the structural rigidity, specifically for $\vartheta=0.05$. One may reason that for smaller structural rigidity, wave loads on the flexible barrier can cause it to deflect to a larger 


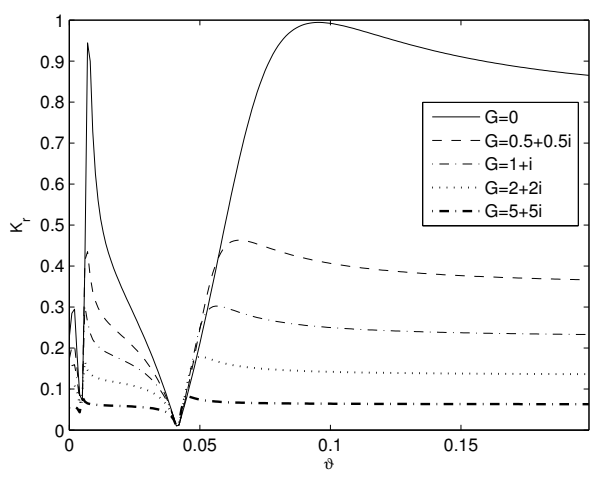

(a)

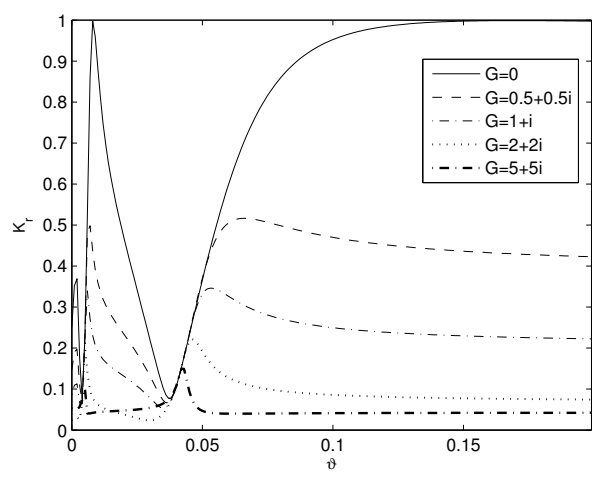

(b)

Fig. 13. Effect of the porous-effect parameter $G$ on the reflection coefficient as a function of the non-dimensional flexural rigidity $\vartheta$. In (a) $h_{2} / h_{1}=1$, and (b) $h_{2} / h_{1}=0.5$, with $\theta=30^{\circ}$ and $h_{3} / h_{1}=1.4$.

extent. Further, Fig. 13(a) depicts that nearly full reflection occurs near $\vartheta=0.09$ in the case of a uniform depth for $G=0$. However, full reflection occurs for $\vartheta \geq 0.14$ in the case of a step-type bed as shown in Fig. 13(b). Moreover, irrespective of the values of the porous-effect parameter $G$, in the case of wave motion over uniform depth in the presence of a depression, minima in wave reflection occur for the same structural rigidity $\vartheta$, while for wave motion in the presence of a step having a depression, minima in wave reflection occur for different values of structural rigidity $\vartheta$. Thus, Figs. 13(a) and 13(b) suggest that, for some particular values of the depth profile, wave and structural parameters, full wave reflection can be achieved by a partial bottom-standing flexible barrier.

\subsection{Bed profile type 3}

Here, the bed profile as shown in Fig. $2(\mathrm{~d})$ is considered, with the bed function $h(x)$ given by

$$
\begin{aligned}
h(x)=h_{2}+\tilde{b}\left[1+2\left(x / L_{0}\right)^{3}\right. & -3\left(x / L_{0}\right)^{2}-(d / \tilde{b})\left\{\sin \left(2 m_{1} \pi x / L_{0}\right)\right. \\
& \left.\left.+\sin \left(2 m_{2} \pi x / L_{0}\right)\right\}\right], 0<x<L_{0},
\end{aligned}
$$

where $d$ is the amplitude of the ripples with $m_{1}$ and $m_{2}$ being the number of ripples. To validate our results, we show in Figs. 14(a) and 14(b) results for the reflection coefficient versus $2 l / \lambda_{1}$ in the absence of the barrier with $L_{0}=m_{1} l, m_{2}=0$ and $h_{2} / h_{1}=1$. Fig. 14(a) shows that the present results agree well with the experimental results by Davies and Heathershaw [1984] and the theoretical results of Chamberlain and Porter [1995]. From these figures, it is observed that due to the resonant interaction between the incoming waves and the periodic sea bottom, significant reflection 
occurs when $2 l / \lambda_{1}=1$. Fig. 14(b) depicts that the Bragg reflection increases as the amplitude of the ripples increases.

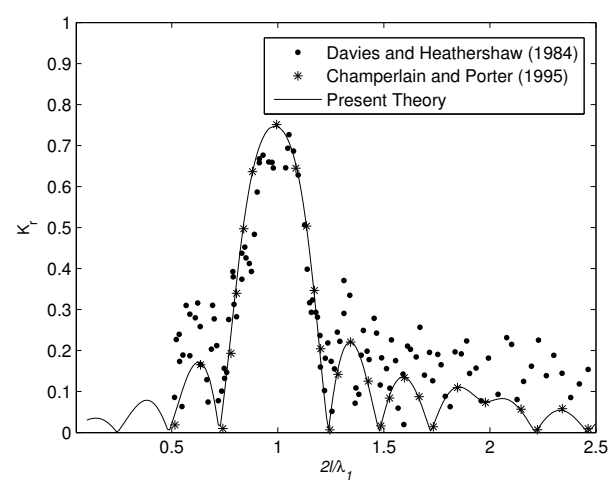

(a)

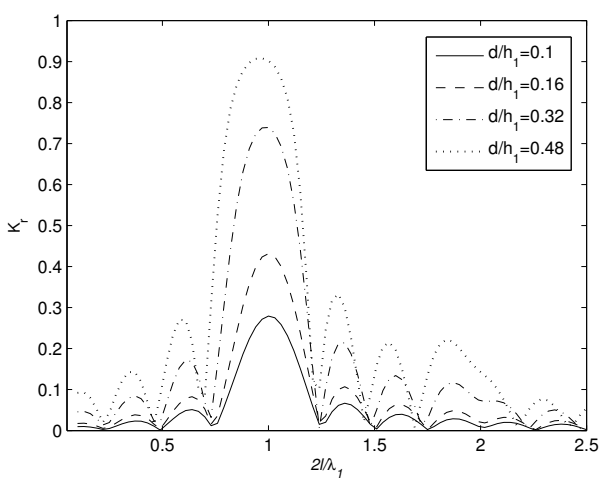

(b)

Fig. 14. The reflection coefficient as a function of $2 l / \lambda_{1}$ : (a) a comparison with the results of Davies and Heathershaw [1984] and Chamberlain and Porter [1995], for $d / h_{1}=0.32$, and (b) effect of the amplitude of the ripples $d / h_{1}$ in the absence of the porous barrier with $h_{2} / h_{1}=1, m_{1}=4, m_{2}=0$, and $\theta=0^{\circ}$.

In Figs. 15(a) and 15(b), the reflection coefficient $K_{r}$ as a function of the nondimensional wave number $k_{0} h_{1}$ in the case of a (a) uniform depth, and (b) step-type bed with $h_{2} / h_{1}=0.5$ are shown for different values of the step length $L_{0} / h_{1}$, respectively, in the absence of the barrier. The figures reveal that the reflection coefficient decreases with an increase in the step length $L_{0} / h_{1}$. Since an increase in step length is related to a decrease in the incline of the step, the decrease in wave reflection may be due to the transmission of more wave energy over the step having a smaller slope. Further, the number of optima in wave reflection increases with an increase in the horizontal step length $L_{0} / h_{1}$. The figures also reveal that, for $k_{0} h_{1} \approx 0$, zero reflection occurs over a topography of sinusoidal profile in an otherwise uniform water depth,while non-zero reflection occurs for a step-type bottom. Moreover, Bragg resonance occurs for larger values of $L_{0} / h_{1}$ in the case of uniform depth. In contrast, Bragg resonance does not occur in the case of a step of sinusoidal profile.

In Figs. 16(a) and 16(b), the reflection coefficient $K_{r}$ versus the non-dimensional wave number $k_{0} h_{1}$ are shown for different values of (a) depth ratio $h_{2} / h_{1}$, and (b) amplitude of the ripple $d / h_{1}$, respectively, in the case of wave scattering by a bottomstanding partial flexible barrier in the presence of a sinusoidal step. Fig. 16(a) reveals that wave reflection increases with an increase in step height. Fig. 16(b) shows that the amplitude of the oscillatory pattern in wave reflection increases with an increase in the amplitude of the ripples $d / h_{1}$ for deep water waves, while wave reflection is independent of the ripple amplitude $d / h_{1}$ for long waves. 


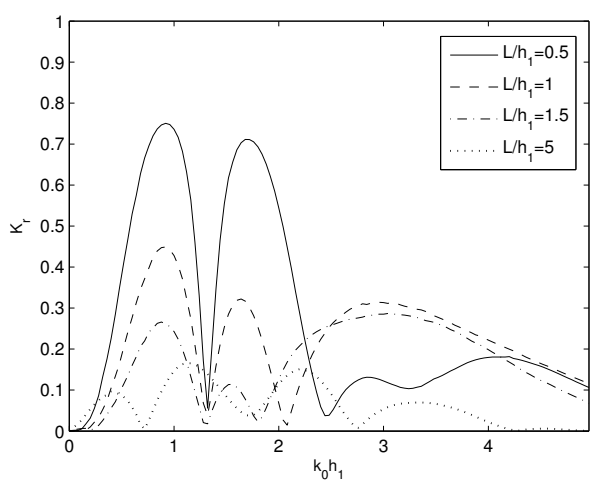

(a)

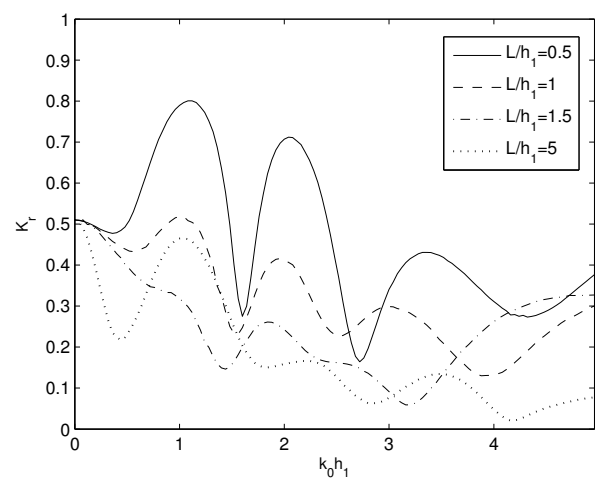

(b)

Fig. 15. Effect of the step length $L_{0} / h_{1}$ on the reflection coefficient as a function of the nondimensional incident wave number $k_{0} h_{1}$ in the absence of the porous barrier. In (a) $h_{2} / h_{1}=1$, and (b) $h_{2} / h_{1}=0.5$, with $m_{1}=5, m_{2}=2, \theta=30^{\circ}$, and $d / h_{1}=0.16$.

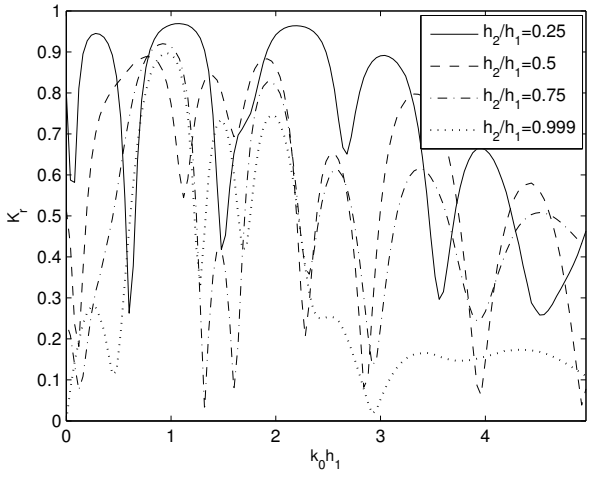

(a)

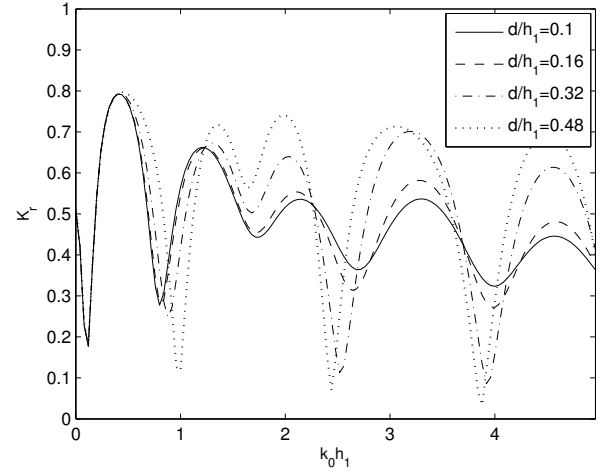

(b)

Fig. 16. Effect of the (a) depth ratio $h_{2} / h_{1}$, and (b) amplitude of the ripple $d / h_{1}$ on the reflection coefficient as a function of the non-dimensional wave number $k_{0} h_{1}$. In (a) $d / h_{1}=0.16$, and (b) $d / h_{1}$, with $h_{2} / h_{1}=0.5, m_{1}=5, m_{2}=2, \theta=30^{\circ}$, and $G=0.5+0.5 \mathrm{i}$.

In Figs. 17(a) and 17(b), the reflection coefficient versus the normalized distance between the porous barrier and step $L_{1} / \lambda_{1}$ are plotted for different values of (a) porous-effect parameter $G$ and (b) step length $L_{0} / h_{1}$ respectively for a bottomstanding flexible porous barrier. Both the figures reveal that the general pattern of wave reflection changes periodically with an increase in $L_{1} / h_{1}$ which is similar to Figs. 12(a) and 12(b). However, minor changes in the periodicity of wave reflection occurs with the change in the absolute value of $G$ and $L_{0} / h_{1}$, which can be caused by a change of the phase of the reflected wave. Further, the magnitude of the maxima 


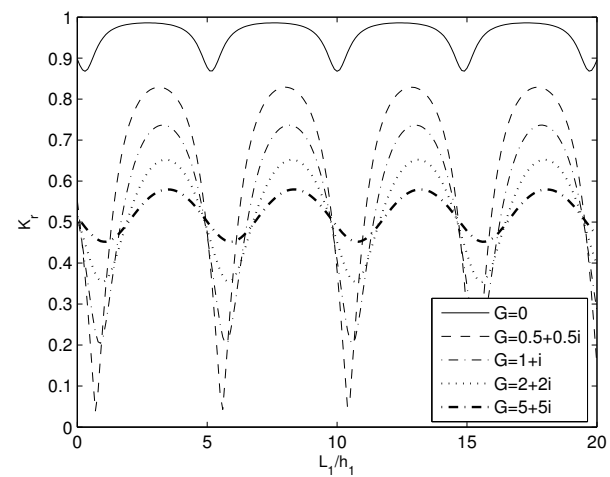

(a)

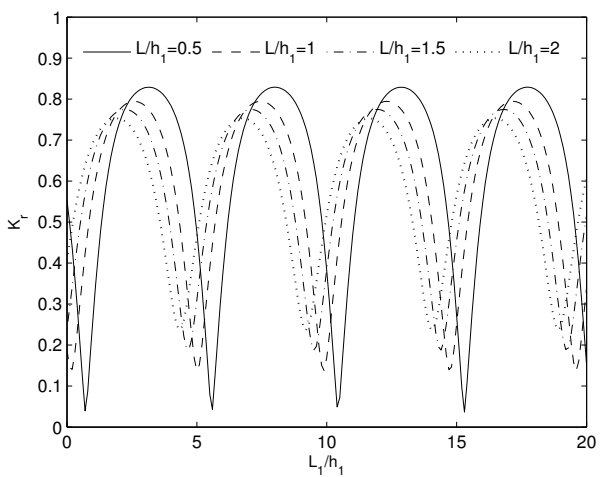

(b)

Fig. 17. Effect of the (a) porous-effect parameter $G$, and (b) step length $L_{0} / h_{1}$ on the reflection coefficient as a function of the normalized distance between the porous barrier and step $L_{1} / \lambda_{1}$. In (a) $L_{0} / h_{1}=0.5$, and (b) $G=0.5+0.5 \mathrm{i}$, with $m_{1}=5, m_{2}=2, d / h_{1}=0.16$, and $\theta=30^{\circ}$.

in the wave reflection decreases with an increase in the absolute value of the porouseffect parameter $G$ and step length $L_{0} / h_{1}$. However, opposite trends are observed for minima in the wave reflection.

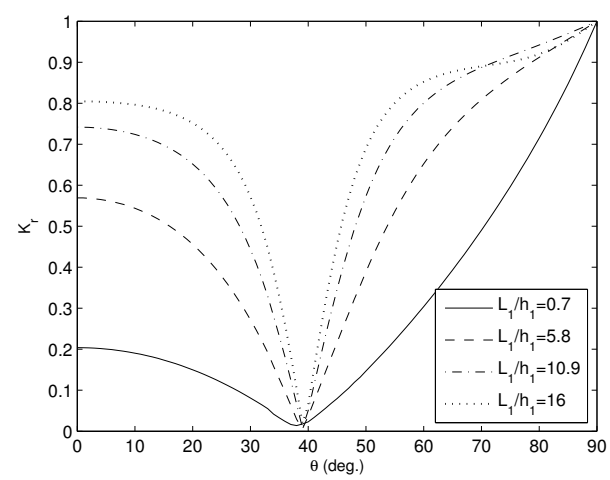

(a)

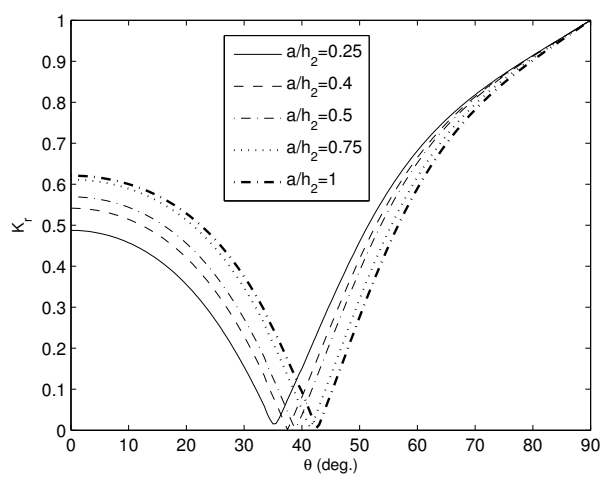

(b)

Fig. 18. Effect of the (a) normalized distance between the porous barrier and step $L_{1} / \lambda_{1}$, and (b) length of the surface-piercing barrier $a / h_{2}$ on the reflection coefficient as a function of the incident angle $\theta$. In (a) $a / h_{2}=0.5$, and (b) $a / h_{2}$, with $L_{1} / h_{1}=5.8$, and $k_{0} h_{1}=0.5, m_{1}=5, m_{2}=2$, $d / h_{1}=0.15$, and $G=0.5+0.5 \mathrm{i}$.

In Figs. 18(a) and 18(b), the reflection coefficient versus the incident angle $\theta$ are plotted for different values of (a) normalized distance between the porous barrier and step $L_{1} / \lambda_{1}$ and (b) length of the surface-piercing flexible porous barrier $a / h_{2}$ 


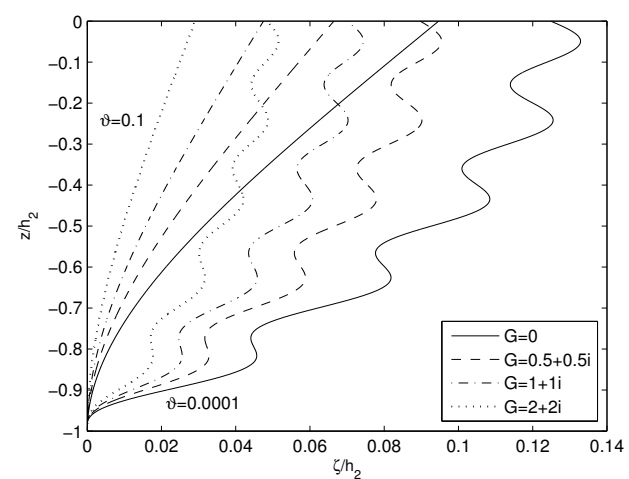

(a)

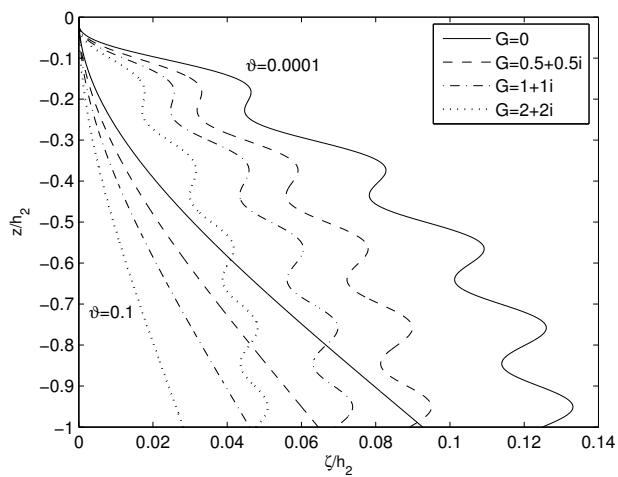

(b)

Fig. 19. Effect of the porous-effect parameter $G$ on the plate deflection of a (a) bottom-standing, and (b) surface-piercing barrier, for $\vartheta=0.1$ and 0.0001 with $L_{1} / h_{1}=3, k_{0} h_{1}=0.5, m_{1}=5$, $m_{2}=2, d / h_{1}=0.15$ and $a / h_{2}=1$.

respectively. Both figures reveal that with an increase in the angle of incidence $\theta$, the wave reflection decreases until reaching a minimum before it increases again. Fig. 18(a) reveals that nearly zero reflection occurs periodically for $L_{1} / h_{1}=5.1$ and $\theta \approx 39^{\circ}$, which is referred to as the critical angle and is denoted as $\theta_{c}$. Further, the reflection coefficient increases with a periodic change in the horizontal distance $L_{1} / h_{1}$. Furthermore, Fig. 18(b) shows that the wave reflection increases with an increase in the length of the barrier $a / h_{2}$ for $0<\theta<\theta_{c}$. However, an opposite trend is observed for $\theta>\theta_{c}$. For $a / h_{2} \geq 0.4$, the wave reflection becomes nearly zero minimum in the range of $39^{\circ}-44^{\circ}$. Moreover, there is a right shift in the wave reflection with an increase in $a / h_{2}$ which may be due to the change of phase of the reflected wave.

Figs. 19(a) and 19(b) show the deflection profiles of a (a) bottom-standing, and (b) surface-piercing flexible porous barrier for various values of the porous-effect parameter $G$. From these figures, it is clearly seen that the deflection of the flexible barrier decreases with an increase in the absolute value of the porous-effect parameter $G$. Further, the barrier deflection increases with an increase in the structural rigidity $\vartheta$. Similar observation has been made previously by Koley et al. [2015a]. Moreover, Fig. 19(a) confirms that zero deflection occurs at the bottom for a bottom-standing porous barrier, as is expected. It is also seen from the figure that the barrier deflection is larger near the submerged free end. On the other hand, for a surface-piercing porous barrier which is assumed to be fixed at the free surface and free at the submerged end, an opposite trend is observed for the barrier deflection. 


\section{Conclusion}

We have presented a study on oblique wave scattering by partial flexible porous barriers in the presence of a step-type bottom of various profiles. Both the cases of bottom-standing and surface-piercing barrier configurations are considered, and three types of bed function $h(x)$ are used to describe the profile of the stepped bottom: (i) sloping step, (ii) step having a protrusion/depression, and (iii) double sinusoidal step. The mathematical problems are solved for the physical variables using the methods of mild-slope and least-squares approximation. The effectiveness of the bottom-standing and surface-piercing barriers has been determined by looking into the reflection/transmission coefficients and barrier deflections for various physical parameters associated with the wave and structure. The computed results are validated by comparison with known results available in the literature for different cases. The study reveals that for some particular values of the bed profiles, wave and structural parameters, nearly zero/full reflection occurs. In particular, in the case of a sloping step, zero wave reflection will happen at $\theta=41^{\circ}$ for $G=5+5 \mathrm{i}$. Further, for a bottom-standing partial flexible porous barrier, full reflection occurs for $G=0$ with $k_{0} h_{1}=0.4$, while nearly zero reflection occurs for $G=5+5 \mathrm{i}$ with $k_{0} h_{1}=1.3$. Also, in the case of a step having a depression, nearly full/zero reflection occurs. For a step of sinusoidal profile, nearly zero reflection occurs periodically when $L_{1} / h_{1}=5.1$ at $\theta \approx 39^{\circ}$, while for $a / h_{2} \geq 0.4$, the minimum wave reflection becomes nearly zero in the range of $39^{\circ}-44^{\circ}$. Moreover, the occurrence of Bragg resonance has been examined for a step-type bed in the presence/absence of flexible porous barriers. These findings should promote a better understanding of how flexible porous barriers over a step-type bed may function to dissipate wave energy to create a zone of tranquility in the marine environment.

\section{References}

Abul-Azm, A. [1994] Diffraction through wide submerged breakwaters under oblique waves, Ocean Engineering 21(7), 683-706.

Behera, H., Kaligatla, R. and Sahoo, T. [2015] Wave trapping by porous barrier in the presence of step type bottom, Wave Motion 57, 219-230.

Behera, H., Mandal, S. and Sahoo, T. [2013] Oblique wave trapping by porous and flexible structures in a two-layer fluid, Physics of Fluids 25(11), 112110.

Behera, H. and Sahoo, T. [2014] Gravity wave interaction with porous structures in two-layer fluid, Journal of Engineering Mathematics 87(1), 73-97.

Bennetts, L., Biggs, N. and Porter, D. [2009] The interaction of flexural-gravity waves with periodic geometries, Wave Motion 46(1), 57-73.

Berkhoff, J. C. W. [1973] Computation of combined refraction-diffraction, Proc. of 13th Int'l Conf. on Coastal Eng, Vancouver, Canada, ASCE. , 471-490.

Bhattacharjee, J. and Guedes Soares, C. [2011] Oblique wave interaction with a floating structure near a wall with stepped bottom, Ocean Eng. 38, 1528-1544. 
Chamberlain, P. and Porter, D. [1995] The modified mild-slope equation, Journal of Fluid Mechanics 291, 393-407.

Davies, A. and Heathershaw, A. [1984] Surface-wave propagation over sinusoidally varying topography, Journal of Fluid Mechanics 144, 419-443.

Dean, W. [1945] On the reflection of surface waves by a submerged plane barrier, Grt. Brit. Ministry of Supply, Wave Report $\mathbf{8}$.

Huang, Z., Li, Y. and Liu, Y. [2011] Hydraulic performance and wave loadings of perforated/slotted coastal structures: a review, Ocean Engineering 38(10), 10311053.

Isaacson, M., Premasiri, S. and Yang, G. [1998] Wave interactions with vertical slotted barrier, Journal of Waterway, Port, Coastal, and Ocean Engineering 124(3), 118-126.

Karmakar, D., Bhattacharjee, J. and Soares, C. G. [2013] Scattering of gravity waves by multiple surface-piercing floating membrane, Applied Ocean Research 39, 4052.

Karmakar, D. and Soares, C. G. [2014] Wave transformation due to multiple bottomstanding porous barriers, Ocean Engineering 80, 50-63.

Koley, S., Kaligatla, R. and Sahoo, T. [2015a] Oblique wave scattering by a vertical flexible porous plate, Studies in Applied Mathematics 135(1), 1-34.

Koley, S., Sarkar, A. and Sahoo, T. [2015b] Interaction of gravity waves with bottomstanding submerged structures having perforated outer-layer placed on a sloping bed, Applied Ocean Research 52, 245-260.

Li, Y., Liu, Y. and Teng, B. [2006] Porous effect parameter of thin permeable plates, Coastal Engineering Journal 48(04), 309-336.

Losada, I., Silva, R. and Losada, M. [1996] 3-d non-breaking regular wave interaction with submerged breakwaters, Coastal Engineering 28(1), 229-248.

Manam, S. and Kaligatla, R. [2012] A mild-slope model for membrane-coupled gravity waves, Journal of Fluids and Structures 30, 173-187.

Mandal, B. and Chakrabarti, A. [2000] Water wave scattering by barriers (Wit Pr/Computational Mechanics).

Mohapatra, S., Ghoshal, R. and Sahoo, T. [2013] Effect of compression on wave diffraction by a floating elastic plate, Journal of Fluids and Structures 36, 124135 .

Penney, W. and Price, A. T. [1952] Part i. the diffraction theory of sea waves and the shelter afforded by breakwaters, Philosophical Transactions of the Royal Society of London A: Mathematical, Physical and Engineering Sciences 244(882), 236-253.

Porter, D. and Staziker, D. [1995] Extensions of the mild-slope equation, Journal of Fluid Mechanics 300, 367-382.

Sahoo, T., Chan, A. and Chwang, A. [2000] Scattering of oblique surface waves by permeable barriers, Journal of waterway, port, coastal, and ocean engineering 126(4), 196-205.

Smith, R. and Sprinks, T. [1975] Scattering of surface waves by a conical island, 
Journal of Fluid Mechanics 72(02), 373-384.

Suh, K. D. and Park, W. S. [1995] Wave reflection from perforated-wall caisson breakwaters, Coastal Engineering 26(3), 177-193.

Toledo, Y. and Agnon, Y. [2011] Three dimensional application of the complementary mild-slope equation, Coastal Engineering 58(1), 1-8.

Wang, K.-H. and Ren, X. [1993] Water waves on flexible and porous breakwaters, Journal of Engineering Mechanics 119(5), 1025-1047.

Wiegel, R. L. [1964] Oceanographical engineering (Prentice-Hall, Englewood Cliffs, NJ, USA).

Yip, T. L., Sahoo, T. and Chwang, A. T. [2002] Trapping of surface waves by porous and flexible structures, Wave Motion 35(1), 41-54. 